\title{
Association between high levels of Notch3 expression and high invasion and poor overall survival rates in pancreatic ductal adenocarcinoma
}

\author{
JIN-XUE ZHOU ${ }^{1 *}$, LIANG ZHOU ${ }^{2 *}$, QING-JUN LI ${ }^{1 *}$, WEN FENG ${ }^{1 *}$, PEI-MIN WANG ${ }^{4 *}$, ER-FENG LI $^{2}$, \\ WEN-JING GONG ${ }^{2}$, MING-WEN KOU ${ }^{2}$, WEI-TING GOU ${ }^{2}$ and YAN-LING YANG ${ }^{3}$ \\ ${ }^{1}$ Department of Hepatobiliary and Pancreatic Surgery, Henan Tumor Hospital, Zhengzhou, Henan 450008; \\ ${ }^{2}$ Department of General Surgery, The 155 Central Hospital of PLA, Kaifeng, Henan 471000; \\ ${ }^{3}$ Department of Hepatobiliary Surgery, Xijing Hospital, The Fourth Military Medical University, Xi'an, Shannxi 710032; \\ ${ }^{4}$ Department of General Surgery, First People's Hospital, Xiang Xin, Henan 453000, P.R. China
}

Received March 26, 2016; Accepted August 11, 2016

DOI: 10.3892/or.2016.5079

\begin{abstract}
Pancreatic ductal adenocarcinoma (PDAC) is a commonly fatal tumour. It is characterized by early metastasis and high mortality. Many patients die as a result of PDAC tumour progression. However, the underlying mechanism of invasion and metastasis in PDAC is still not fully understood. Previous studies showed that the Notch signalling pathway may play an important role in the progression of tumour invasion and metastasis. However, it is not yet known whether the Notch signalling pathway participates in the progression of invasion in PDAC. In the present study, immunohistochemistry showed that a high expression of Notch3 was correlated with tumour grade, metastasis, venous invasion and American Joint Committee on Cancer (AJCC) tumor-node-metastasis (TNM) stage. Kaplan-Meier curves suggested that a high expression of Notch3 was a significant risk factor for shortened survival time. We also showed that inhibition of Notch3 had an anti-invasion role in PDAC cells. In vitro, the inhibition of Notch 3 reduced the migration and invasion capabilities of PDAC cells by regulating the expressions of E-cadherin, CD44v6, MMP-2, MMP-9, VEGF and uPA via regulating the COX-2 and ERK1/2 pathways. These results indicated that
\end{abstract}

Correspondence to: Professor Liang Zhou, Department of General Surgery, The 155 Central Hospital of PLA, Kaifeng, Henan 471000, P.R. China

E-mail: zhouliang_004@126.com

Professor Yan-Ling Yang, Department of Hepatobiliary Surgery, Xijing Hospital, The Fourth Military Medical University, Xi'an, Shannxi 710032, P.R. China

E-mail: 1121050538@qq.com

*Contributed equally

Key words: pancreatic ductal adenocarcinoma, Notch3, invasion, metastasis, overall survival, clinicopathological correlation downregulation of the Notch signalling pathway may be a novel and useful approach for preventing and treating PDAC invasion.

\section{Introduction}

As a highly aggressive malignant disease, pancreatic ductal adenocarcinomax (PDAC) is the fourth leading cause of cancer-related death and has a median survival of 6 months (1). Despite extensive clinical efforts, the mortality of patients with PDAC has not significantly altered, and the 5-year survival rate remains unacceptably low. During the diagnosis of pancreatic cancer, early metastasis is often found, eliminating the option of curative surgery (2-4). However, in clinical practice, few markers other than tumor-node-metastasis (TNM) stage can be used as independent prognostic factors of tumour progression. Moreover, the molecules involved in the progression of metastasis may be markers for the early detection of recurrence or metastasis as well as prognostic indicators for surgical intervention. Therefore, it is necessary to further explore new indicators for the prediction and evaluation of tumour progression and patient prognosis.

As an evolutionarily conserved signalling pathway, the Notch signalling pathway has been shown involved in cell-fate determination, tissue patterning and morphogenesis, and cell differentiation, proliferation and death $(5,6)$. Since the Notch signalling pathway participates in progression processes such as proliferation and apoptosis, it may be associated with tumourigenesis (7). Previous studies indicated that many signalling pathways, including the Notch signalling pathway, may play an important role in PDAC (8). The Notch signalling pathway plays a critical role in the control of cell proliferation, differentiation, apoptosis, invasion and metastasis in PDAC (9). Studies frequently show that the expression of Notch receptors and their ligands increase in PDAC (10). Inhibition of Notch1 is the main cause of decreased proliferation, migration, and invasion and increased apoptosis in PDAC cells $(11,12)$. In an in vivo experiment, downregulation of the Notch signalling pathway led to inhibition of the canceration and development 
of PDAC cells (13). Contradictorily, the Notch signalling pathway may play an oncogenic or onco-suppressive role in tumourigenesis, and its function is also context-dependent in PDAC (14). Surprisingly, Notch1 may have an onco-suppressive role in $K$-ras-induced PDAC (15). All of these findings indicate that further research is needed to explore the role of the Notch signalling pathway in PDAC since the relationship between the expression of Notch 3 and survival in PDAC patients is unclear.

In the present study, we used immunohistochemistry to investigate the protein expression of Notch3. This is the first study to explore the potential relationship between the protein expression of Notch3 and the prognosis of patients with PDAC. Furthermore, we also explored the role of Notch3 in the migration and invasion of PDAC.

\section{Materials and methods}

Patients and tissue specimens. We collected PDAC and adjacent non-cancerous tissues (at least $1.5 \mathrm{~cm}$ from the tumour) from 101 patients who underwent surgery for primary PDAC at the Department of Hepatobiliary Surgery at Xijing Hospital (Xi'an, China) between 2002 and 2010. These patients had not received preoperative treatments such as chemotherapy, ethanol injection or transarterial chemoembolization. A total of 59 male and 42 female patients participated in the present study. The median age of the patients was 55.3 years (range, 39-82 years). Our research was approved by the Ethics Committee of the Fourth Military Medical University and conformed to the ethical guidelines of the 2004 Declaration of Helsinki. Written informed consent was obtained from each patient or from his/her legal guardian. To ensure the validity of the experiment, histopathologic examinations were performed to confirm that the tumour samples contained an adequate number of cancer cells and that no cancer cells had contaminated the non-cancerous tissues. All specimens were fixed in $10 \%$ formalin and embedded in paraffin, and $4-\mu \mathrm{m}$ serial sections were examined using immunohistochemistry. We assessed clinical parameters such as gender, age, tumour grade, metastasis and American Joint Committee on Cancer (AJCC) TNM stage. Of the 38 cases diagnosed with metastasis, 26 had venous invasion, 11 had bile duct tumour thrombi, and 17 had lymph node metastasis. A 1-year follow-up study was conducted to perform survival calculations for these patients.

Cell culture and reagents. The human pancreatic non-tumour cell line (HPDE6c7) and human PDAC cell lines (ASPC-1, BXPC-3, CFPAC-1 and PANC-1) cultivated in Dulbecco's modified Eagle's medium (DMEM) supplemented with 10\% foetal calf serum (Sigma Chemical Co., St. Louis, MO, USA). The PDAC cells were seeded into 6-well cell culture plates at a density of $1 \times 10^{5}$ cells/well. Primary antibodies against Notch3, CD44v6, E-cadherin, matrix metalloproteinase-2 (MMP-2), MMP-9, vascular endothelial growth factor (VEGF), urokinase-type plasminogen activator (uPA), cyclooxygenase-2 (COX-2), extracellular signal-regulated kinase 1 and 2 (ERK1/2), p-ERK1/2 and GAPDH were purchased from Santa Cruz Biotechnology (Santa Cruz, CA, USA). All secondary antibodies were obtained from Pierce (Rockford, IL, USA). Notch3 small interfering RNA (siRNA) and siRNA controls were obtained from Santa Cruz Biotechnology.
Lipofectamine 2000 was purchased from Invitrogen (Carlsbad, CA, USA). All other chemicals and solutions were purchased from Sigma-Aldrich unless otherwise indicated.

Immunohistochemistry and evaluation of staining. The avidin-biotin-peroxidase method was used to perform immunohistochemistry of all tissues. Xylene was used to deparaffinise the sections, and a graded alcohol series was used to dehydrate prior to blocking in endogenous peroxidase activity using $0.5 \% \mathrm{H}_{2} \mathrm{O}_{2}$ in methanol for $10 \mathrm{~min}$. The sections were incubated with $10 \%$ normal goat serum in phosphate-buffered saline (PBS) for $1 \mathrm{~h}$ at room temperature to block non-specific binding. Without washing, the sections were incubated in PBS with an anti-Notch3 antibody (1:50) at $4^{\circ} \mathrm{C}$ overnight in a humidified chamber. Then, the sections were incubated with biotinylated IgG (1:200; Sigma) for $2 \mathrm{~h}$ at room temperature. A streptavidin-peroxidase complex was used for detection. The brown colour indicative of peroxidase activity was obtained by incubating with $0.1 \%$ 3,3-diaminobenzidine (Sigma) in PBS with $0.03 \% \mathrm{H}_{2} \mathrm{O}_{2}$ for $10 \mathrm{~min}$ at room temperature. Using a previously described immunoreactivity scoring system, two pathologists who were blinded to the clinicopathological results, and patient outcome independently scored the tissue specimens (16). Based on the score, all PDAC specimens were divided into two subgroups: the low-expression group (score of 0-4) and the high-expression group (score of 5-12).

Small interfering RNA transfection. Either Notch3 siRNA or control siRNA were transfected into BXPC-3 and PANC-1 cells using Lipofectamine 2000 according to the manufacturer's protocol. The siRNA-treated cells were seeded into 6 -well cell culture plates at a density of $1 \times 10^{5}$ cells/well. The cells grew for an additional $24 \mathrm{~h}$, and were then harvested for further analysis.

Real-time reverse transcription-PCR. Total RNA was extracted and reverse-transcribed. The primers used for the PCR reaction were as follows: Notch3 forward primer (5'-aaggacgtggcctctggt-3'), and reverse primer (5'-tcaggctctca ccettgg-3'); and GAPDH forward primer (5'-AAATCCCATCA CCATCTTCC-3'), and reverse primer (5'-TCACACCCATGA CGAACA-3'). The primer sequences were verified by running a virtual PCR, and the primer concentrations were optimized to prevent primer-dimer formation. Additionally, dissociation curves were evaluated to prevent non-specific amplification. Real-time PCR amplifications were performed using an Mx4000 Multiplex QPCR System (Stratagene, La Jolla, CA, USA) with 2X SYBR-Green PCR Master Mix (Applied Biosystems). Data were analysed according to the comparative $\mathrm{Ct}$ method and were normalized to GAPDH expression in each sample.

Protein extraction and western blotting. The cells were lysed in lysis buffer [50 mmol/l Tris (pH 7.5), $100 \mathrm{mmol} / \mathrm{l} \mathrm{NaCl}$, $1 \mathrm{mmol} / 1$ EDTA, $0.5 \% \mathrm{NP} 40,0.5 \%$ Triton X-100, $2.5 \mathrm{mmol} / \mathrm{l}$ sodium orthovanadate, $10 \mu \mathrm{l} / \mathrm{ml}$ protease inhibitor cocktail and $1 \mathrm{mmol} / \mathrm{l} \mathrm{PMSF}$ ] by incubating for $20 \mathrm{~min}$ at $4^{\circ} \mathrm{C}$. The protein concentration was determined using the Bio-Rad assay system (Bio-Rad, Hercules, CA, USA). Total proteins were 

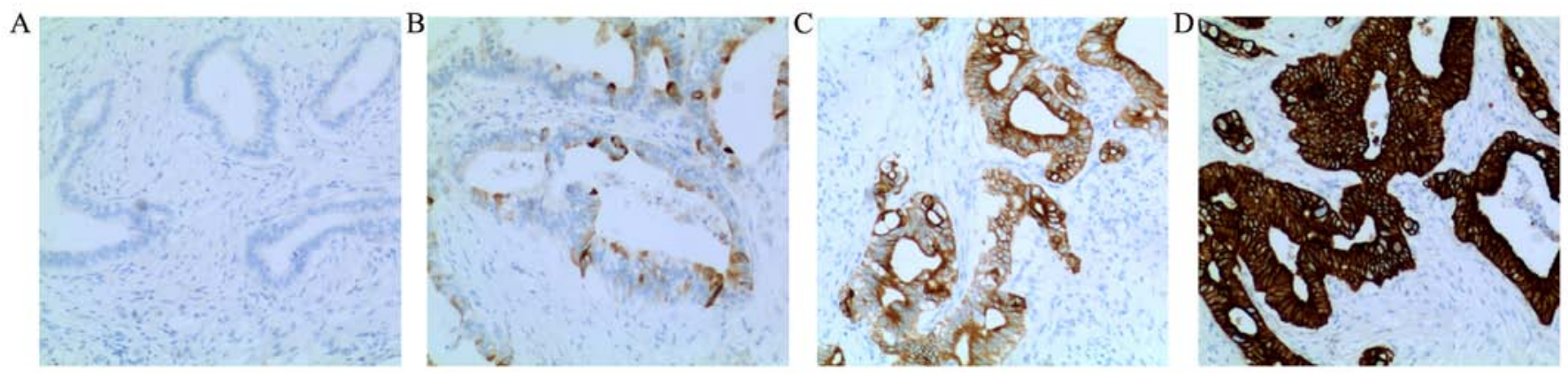

Figure 1. Different levels of Notch3 expression in PDAC tissues. (A) Negative. (B) Weak. (C) Moderate. (D) Strong.

fractionated using SDS-PAGE and transferred onto nitrocellulose membranes. The membranes were blocked with $5 \%$ non-fat dried milk or bovine serum albumin in $1 \mathrm{X}$ TBS buffer containing $0.1 \%$ Tween-20, and then incubated with the appropriate primary antibodies. Horseradish peroxidase-conjugated anti-rabbit or anti-mouse IgG was used as the secondary antibody, and the protein bands were detected using an enhanced chemiluminescence detection system (Amersham Pharmacia Biotech). Quantification of the western blot analyses was performed using laser densitometry, and the relative protein expression was then normalized to GAPDH levels.

MTT assay. Treated cells were seeded into 96-well cell culture plates at a density of $1 \times 10^{4}$ cells/well and incubated for up to 48 h. Using 3-(4,5-dimethyl-2-thiazolyl)-2,5-diphenyl-2H-tetrazolium bromide (MTT) assay (Sigma Chemicals Co.), cell viability was assessed according to the manufacturer's protocols. Each experiment had six replications and was repeated three times. The data are summarized as the means \pm SDs.

Migration and invasion assays. Cell migration and invasion were assessed using non-Matrigel-coated or Matrigel-coated Transwell cell culture chambers $(8-\mu \mathrm{m}$ pore size) (Millipore, Billerica, MA, USA). Briefly, treated cells $\left(5 \times 10^{4}\right.$ cells/well) were serum-starved for $24 \mathrm{~h}$ and plated in the upper insert of a 24-well chamber in serum-free medium. Medium containing $10 \%$ serum as a chemoattractant was added to the well, and the cells were incubated for $24 \mathrm{~h}$. Cells on the upper side of the filters were mechanically removed with a cotton swab. The membrane was fixed with $4 \%$ formaldehyde for $10 \mathrm{~min}$ at room temperature and stained with $0.5 \%$ crystal violet for $10 \mathrm{~min}$. Finally, the number of invasive and migrated cells was counted at a magnification of x 200 in 10 different fields of each filter.

Statistical analysis. Statistical analysis was performed using SPSS 15.0 software (SPSS, Inc., Chicago, IL, USA). Each experiment was repeated at least three times, and all results were summarized and presented as the means \pm SDs. A t-test was used to statistically analyse the differences between means. The $\chi^{2}$ test for proportions was used to analyse the relationship between Notch3 expression and various clinicopathological factors. Survival curves were calculated using the Kaplan-Meier method and compared using the log-rank test. Cox proportional hazard analysis was used for univariate and multivariate analysis to explore the effect of clinicopathological factors and Notch3 expression. P-values $<0.05$ were considered to indicate a statistically significant result.

\section{Results}

Immunohistochemistry of Notch3. Positive staining for Notch 3 was observed mainly in the cytoplasm and at the cell membrane. In adjacent non-cancerous tissues, the expression of Notch3 was weak in the cytoplasm and at the cell membrane. As shown in Fig. 1, the expression of Notch3 was different in PDAC tissue. Negative staining for Notch3 was observed in 15 cases, weak positive staining was observed in 28 cases, moderate positive staining was observed in 23 cases, and strong positive staining was observed in 35 cases.

Relationships between Notch3 expression and clinicopathological characteristics. As pathological factors, gender, age, tumour grade, metastasis and AJCC TNM stage were examined in 101 cases of PDAC. Vascular invasion was also analysed in patients with metastasis. For the present study, the 101 patients were divided into two subgroups: a high-expression group $(n=55)$ and a low-expression group $(n=46)$. The relationships between the expression of Notch3 and the clinicopathological factors are shown in Table I. The results indicate that a high expression of Notch3 was strongly correlated with tumour grade $(\mathrm{P}=0.003)$, metastasis $(\mathrm{P}=0.003)$, venous invasion $(\mathrm{P}=0.008)$ and AJCC TNM stage $(\mathrm{P}<0.001)$. In contrast, a high expression of Notch3 was not correlated with the other pathological factors $(\mathrm{P}>0.05)$. These results suggested that Notch3 may participate in the process of differentiation, invasion and metastasis in PDAC.

Correlation between the expression of Notch3 and the prognosis of PDAC patients. Since the expression of Notch3 was correlated with tumour grade, metastasis, venous invasion and AJCC TNM stage in PDAC, we hypothesized that the expression of Notch3 may have a relationship to the prognosis of patients with PDAC. We used Kaplan-Meier postoperative survival curves to evaluate the correlation between the overall survival rates of patients with PDAC and the expression of Notch3. Log-rank tests showed significantly different survival times between the low and high Notch3 expression groups $(\mathrm{P}<0.001)$. These results suggested that a low expression of Notch3 increases patient survival, whereas a high expression 
Table I. Association of Notch3 expression with clinicopathological factors of the PDAC patients.

\begin{tabular}{|c|c|c|c|c|c|}
\hline \multirow[b]{2}{*}{ Tumour characteristic } & \multirow[b]{2}{*}{$\mathrm{n}$} & \multicolumn{2}{|c|}{ Notch3 } & \multirow[b]{2}{*}{ P-value } & \multirow[b]{2}{*}{$\chi^{2}$} \\
\hline & & $\begin{array}{c}\text { High } \\
(5-12 \text { score, } \%)\end{array}$ & $\begin{array}{c}\text { Low } \\
(0-4 \text { score, } \%)\end{array}$ & & \\
\hline All cases & 101 & $55(54.5)$ & $46(45.5)$ & & \\
\hline \multicolumn{6}{|l|}{ Gender } \\
\hline Male & 59 & $34(57.6)$ & $25(42.4)$ & 0.448 & 0.575 \\
\hline Female & 42 & $21(50.0)$ & $21(50.0)$ & & \\
\hline \multicolumn{6}{|l|}{ Age (years) } \\
\hline$\leq 50$ & 50 & $30(60.0)$ & $20(40.0)$ & 0.268 & 1.227 \\
\hline$>50$ & 51 & $25(49.0)$ & $26(51.0)$ & & \\
\hline \multicolumn{6}{|c|}{ Tumour grade (differentiation) } \\
\hline Well & 33 & $25(75.8)$ & $8(24.2)$ & 0.003 & 8.968 \\
\hline Moderately or poorly & 68 & $30(44.1)$ & $38(55.9)$ & & \\
\hline \multicolumn{6}{|l|}{ Metastasis } \\
\hline Yes & 38 & $28(73.7)$ & $10(26.3)$ & 0.003 & 9.082 \\
\hline No & 63 & $27(42.9)$ & $36(57.1)$ & & \\
\hline \multicolumn{6}{|l|}{ Venous invasion } \\
\hline+ & 26 & $20(76.9)$ & $6(23.1)$ & 0.008 & 7.126 \\
\hline- & 75 & $35(46.7)$ & $40(53.3)$ & & \\
\hline \multicolumn{6}{|l|}{ AJCC TNM stage } \\
\hline I and II & 19 & $1(5.3)$ & $18(74.7)$ & $<0.001$ & 22.834 \\
\hline III and IV & 82 & $54(65.9)$ & $28(34.1)$ & & \\
\hline
\end{tabular}

PDAC, pancreatic ductal adenocarcinoma; AJCC, American Joint Committee on Cancer; TNM, tumor-node-metastasis.

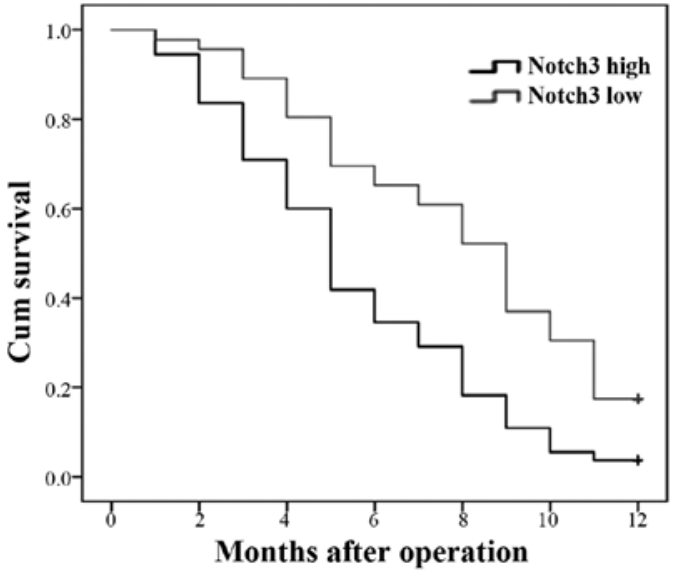

Figure 2. Kaplan-Meier statistical analysis of postoperative survival curves according to Notch3 expression.

of Notch3 reduces patient survival (Fig. 2). The cumulative 1-year survival rate was $17.6 \%$ in patients with a low expression of Notch3, whereas the rate was only $7.1 \%$ in patients with a high expression of Notch3.

The results of the univariate Cox regression analysis showed that metastasis, venous invasion, AJCC TNM stage and the protein expression of Notch3 were strongly correlated with overall survival (2). We also used multivariate Cox
Table II. Univariate and multivariate analysis for overall survival of PDAC patients.

\begin{tabular}{lcc}
\hline Tumour characteristic & Relative risk $(95 \%$ CI $)$ & P-value \\
\hline Univariate & & \\
Gender & $1.024(0.674-1.556)$ & 0.911 \\
Age (years) & $1.075(0.711-1.625)$ & 0.732 \\
Tumour grade & $0.578(0.370-0.902)$ & 0.016 \\
(differentiation) & & \\
Metastasis & $11.292(6.203-20.557)$ & $<0.001$ \\
Venous invasion & $10.904(5.925-20.066)$ & $<0.001$ \\
AJCC TNM stage & $3.694(2.022-6.748)$ & $<0.001$ \\
Notch3 & $2.927(1.876-4.569)$ & $<0.001$ \\
Multivariate & $1.194(0.725-1.965)$ & 0.486 \\
Tumour grade & & \\
(differentiation) & $5.917(2.762-12.676)$ & $<0.001$ \\
Metastasis & $2.516(1.240-5.108)$ & 0.11 \\
Venous invasion & $1.961(0.981-3.920)$ & 0.057 \\
AJCC TNM stage & $1.960(1.181-3.252)$ & 0.009 \\
Notch3 &
\end{tabular}

PDAC, pancreatic ductal adenocarcinoma; 95\% CI, 95\% confidence interval; AJCC, American Joint Committee on Cancer; TNM, tumornode-metastasis. 
A

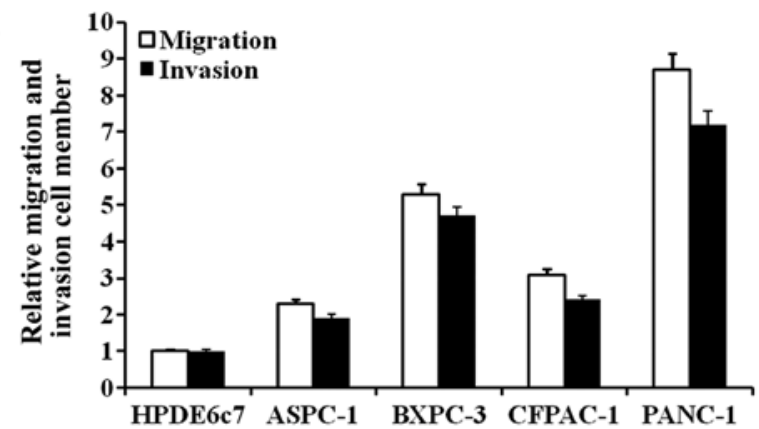

B

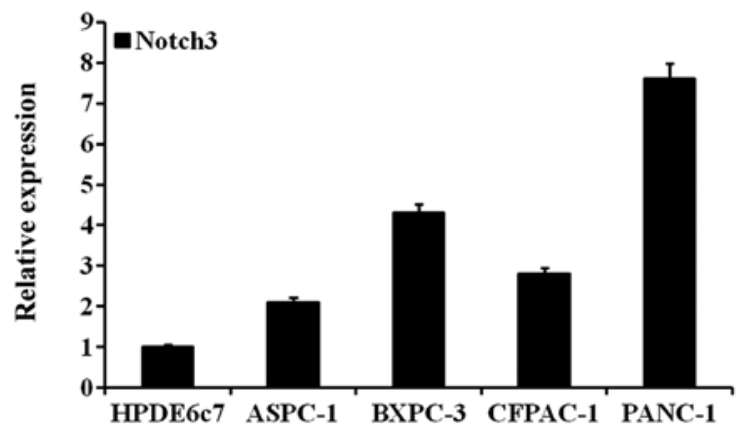

$\mathrm{C}$

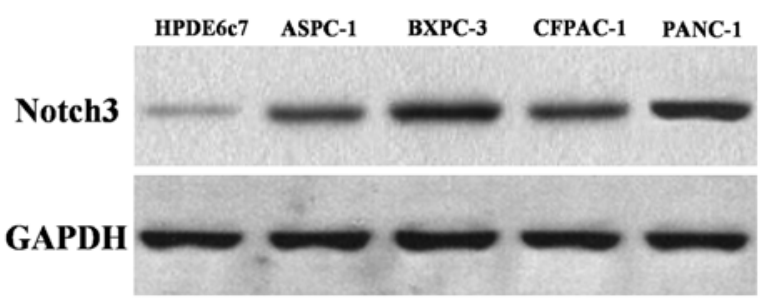

Figure 3. The expression of Notch 3 mRNA and protein in PDAC cells. (A) Using Transwell cell culture chambers, we detected the migration and invasion capabilities of different PDAC cell lines (PDAC cells vs. pancreatic non-tumour cell). (B and C) RT-PCR and western blotting were performed to assess the expression levels of Notch3 in different PDAC cell lines.

regression analysis to evaluate whether the high expression of Notch 3 was an independent predictor of overall survival in patients with PDAC. Data in Table II show that Notch3 expression predicted overall survival in patients with PDAC.

Inhibition of Notch3 decreases PDAC cell migration and invasion capabilities. To assess whether Notch3 participates in PDAC cell invasion and metastasis, we first detected the Notch3 expression in PDAC cells with different migration and invasion capabilities. As shown in Fig. 3A, BXPC-3 and PANC-1 cells had high migration and invasion capabilities. As shown in Fig. 3B and C, as the mRNA and protein expression of Notch3 increased, the migration and invasion capabilities of the PDAC cells tended to increase. In PDAC cells, the expression of Notch 3 mRNA and protein could be effectively inhibited by siRNA (Fig. 4A and B). To detect changes in migration and invasion capabilities, we measured the number of siRNA-transfected PDAC cells using Transwell cell culture chambers. As shown in Fig. 5, the number of BXPC-3 and PANC-1 cells that migrated through the Transwell was significantly lower among the Notch3-inhibited cells than among the control siRNA-transfected cells. According to the MTT assay, inhibition of Notch3 had no effect on cell viability, which

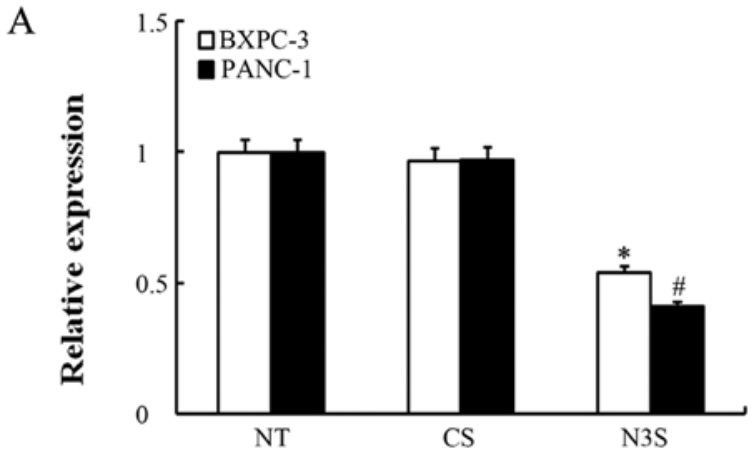

B

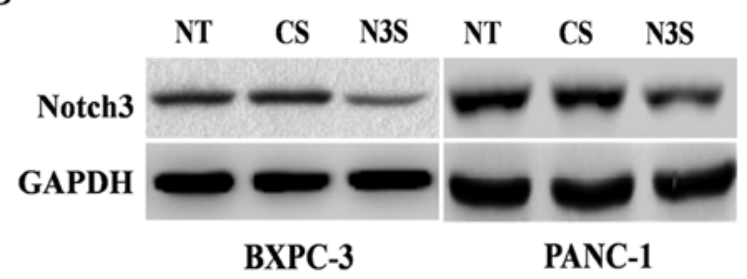

C

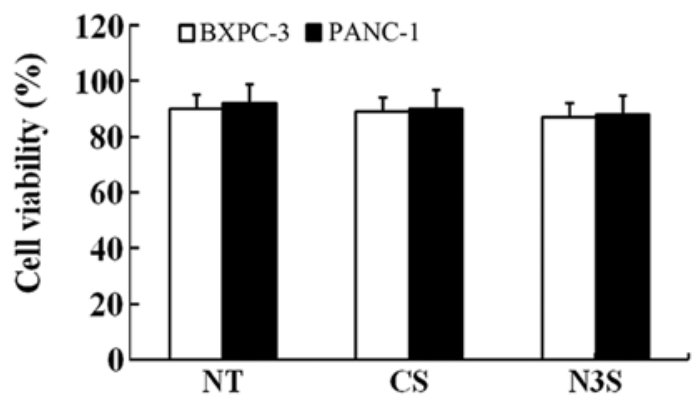

Figure 4. siRNA effectively inhibits the expression of Notch 3 mRNA and protein in PDAC cells. (A and B) RT-PCR and western blotting were performed to assess the expression of Notch3 in three Notch3 siRNA-transfected PDAC cell lines. The expression of Notch3 was normalized to that of GAPDH (Notch3/GAPDH). (C) Viabilities of PDAC cells were measured by MTT. Data represent the mean $\pm \mathrm{SD},{ }^{*} \mathrm{P}<0.05$ compared to control siRNA-transfected BXPC-3 cells; ${ }^{\#} \mathrm{P}<0.05$ compared to control siRNA-transfected PANC-1 cells. NT, non-transfection; N3S, Notch3 siRNA-transfection; CS, control siRNA-transfection.

confirmed that the effects of inhibited Notch3 on cell migration and invasion were independent of apoptosis (Fig. 4C). Thus, based on these results, we can speculate that the inhibition of Notch 3 could decrease the migration and invasion capabilities of PDAC cells.

Inhibition of Notch 3 decreases the protein expression of CD44v6, MMP-2, MMP-9, VEGF and uPA and increase the protein expression of E-cadherin. To explore the potential mechanism of action of Notch3 in PDAC cells, we detected the effect of inhibited Notch 3 on molecules related to metastasis, such as CD44v6, E-cadherin, MMP-2, MMP-9, VEGF and $\mathrm{uPA}$. We found that Notch3 inhibition could decrease the protein expression of CD44v6, MMP-2, MMP-9, VEGF and uPA, whereas the protein expression of E-cadherin increased in PDAC cells with Notch3 inhibition (Fig. 6). These results suggested that Notch3 may be involved in the processes of migration and invasion in PDAC cells by regulating CD44v6, E-cadherin, MMP-2, MMP-9, VEGF and uPA. 
A

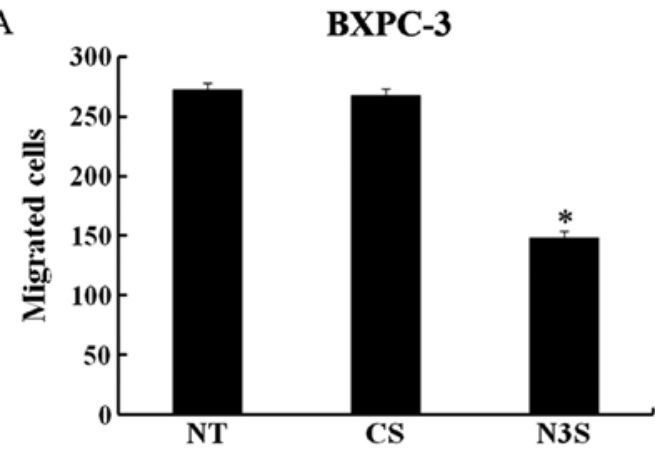

C

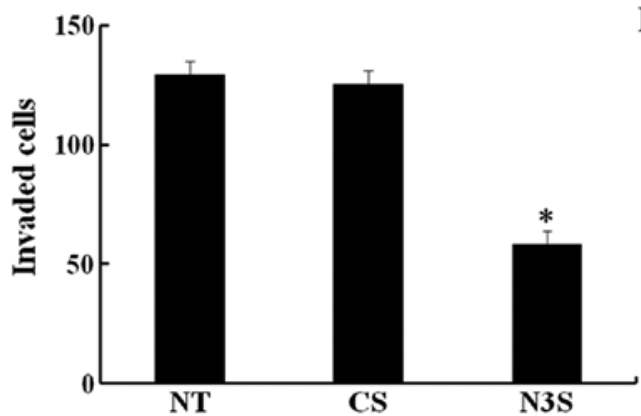

B

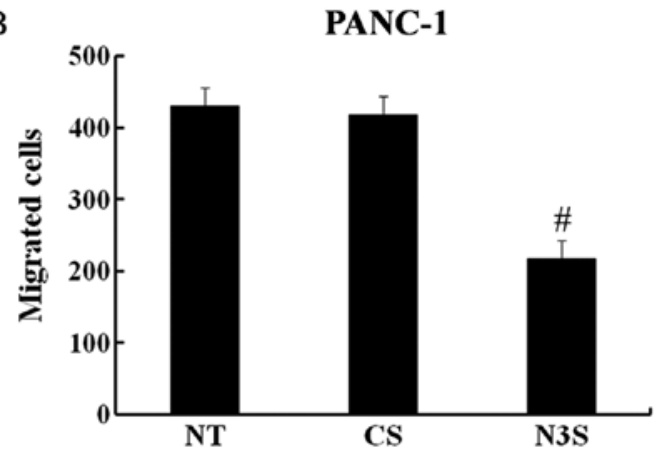

$\mathrm{D}$

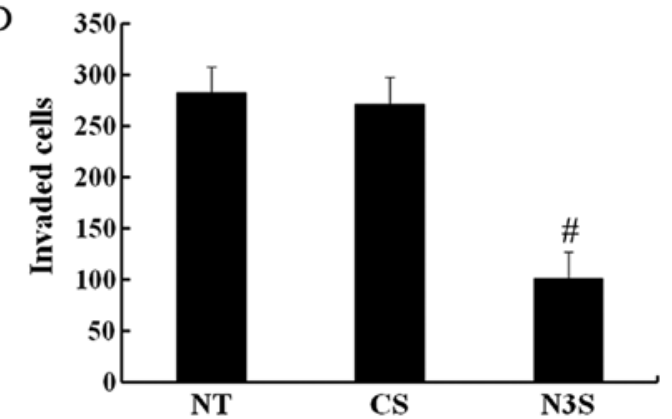

Figure 5. Inhibition of Notch3 by siRNA decreases the in vitro migration (A and B) and invasion (C and D) capabilities of PDAC cells in Transwell assays, compared to control siRNA treatment. The data represent the mean $\pm \mathrm{SD},{ }^{*} \mathrm{P}<0.05$ compared to control siRNA-transfected BXPC-3 cells; ${ }^{*} \mathrm{P}<0.05$ compared to control siRNA-transfected PANC-1 cells. NT, non-transfection; N3S, Notch3 siRNA-transfection; CS, control siRNA-transfection.

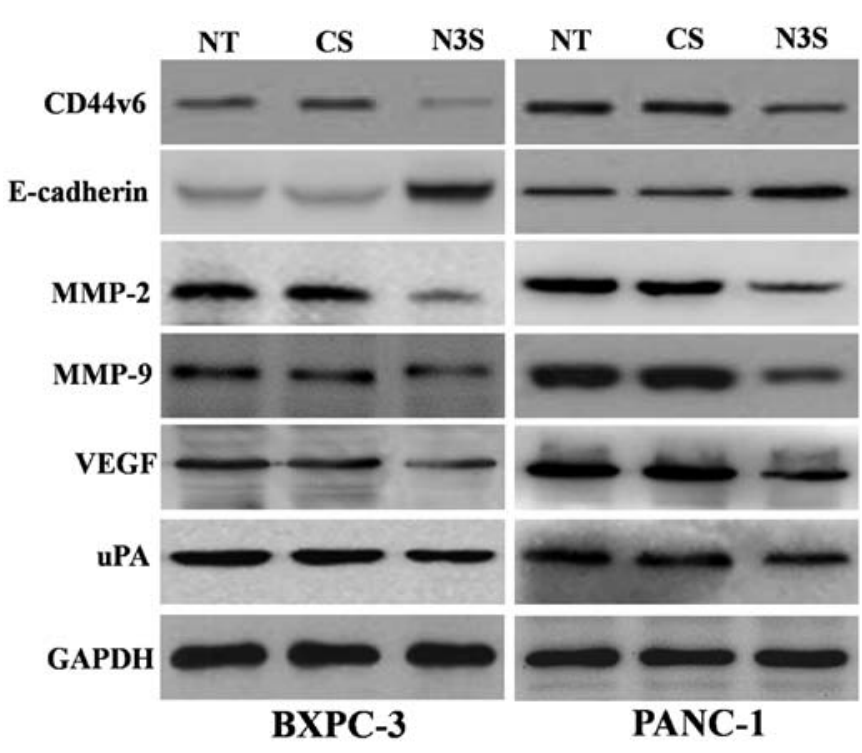

Figure 6. In PDAC cells, the inhibition of Notch3 by siRNA affects the protein expression of CD44v6, E-cadherin, MMP-2, MMP-9, VEGF and uPA. NT, non-transfection; N3S, Notch3 siRNA-transfection; CS, control siRNA-transfection.

Notch 3 regulates the COX-2 and the ERK1/2 pathways. COX-2 is an upstream molecule of CD44v6 and E-cadherin, and the ERK1/2 pathway includes the up-stream molecules of MMP-2, MMP-9, VEGF and uPA. Thus, we explored whether inhibition of Notch 3 could affect the COX-2 and ERK1/2 pathways. In Notch3-inhibited PDAC cells, the expression of COX-2 and p-ERK1/2 decreased, indicating that Notch 3 may be up-stream molecule of COX-2 and the ERK1/2 pathway (Fig. 7).

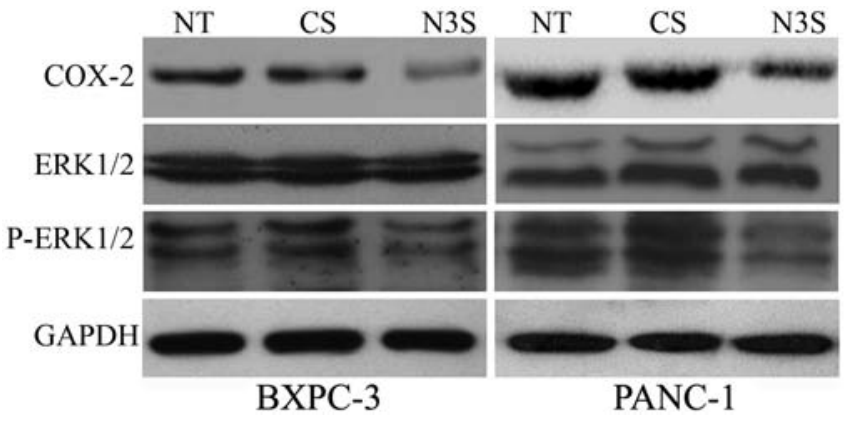

Figure 7. In PDAC cells, the inhibition of Notch3 by siRNA affects the protein expression of COX-2 and ERK1/2. NT, non-transfection; N3S, Notch3 siRNA-transfection; CS, control siRNA-transfection.

\section{Discussion}

The mortality rate of pancreatic ductal adenocarcinoma (PDAC) is high, despite the use of surgery, radiation therapy and chemotherapy to treat PDAC. A lack of effective therapies is the main cause of mortality. However, it is difficult to make an early diagnosis since there are no obvious symptoms and no specific detection methods for the early stage of PDAC, and most patients have lost the chance to undergo radical surgery when the diagnosis is made (1).

Numerous previous studies indicated that the Notch signalling pathway plays an important role in many cellular development processes and can also regulate tumourigenesis $(17,18)$. Although various studies showed that the Notch signalling pathway may play a suppressive role in PDAC in certain specific conditions (15), other studies have indicated that abnormal expression of the Notch signalling pathway may cause tumourigenesis in PDAC (14,19-21). An abnormal 
expression of Notch receptors, Notch ligands and Notch target genes has been observed in PDAC $(10,11,20,21)$. Furthermore, inhibition of the Notch signalling pathway inhibits cell growth, migration and invasion in murine pancreatic cancer cells. However, the role that the Notch pathway plays in PDAC remains unclear.

In the present study, we used immunohistochemistry to examine the expression of Notch3 in PDAC tissues. The results suggested that a high expression of Notch3 was correlated with tumour grade, metastasis, venous invasion and TNM stage. These results strongly indicated that Notch3 may play an important role in the progression of PDAC. An effective prognostic molecular biomarker may be important in evaluating patient status and promoting tumour control. In the present study, the results of survival curves showed that patients with a high expression of Notch3 had a significantly worse overall survival rate (log-rank test; $\mathrm{P}<0.001)$. Previous studies showed that the increased expression of Notch3 in pancreatic cancer was statistically significant for both cytoplasmic and nuclear staining compared to benign tissue (22). One study also showed that cytoplasmic expression of Notch3 was upregulated in tumours in $21 / 35$ patients $(60.0 \%)$ and nuclear Notch 3 was present in 20 resected PDACs (47.6\%) and nuclear Notch3 was associated with the presence of lymph node metastases in resected PDAC specimens (23). In our results, we found positive staining for Notch3 was mainly observed in the cytoplasm and at the cell membrane and nuclear Notch3 was also observed. However, the expression of Notch3 in nuclear was less than the expression of Notch3 in cytoplasm and membrane. These results were similar to previous studies. There may be two reasons for this result. One reason may be that nuclear staining results were different using different antibody which had different protein binding properties and nuclear penetration. Other reason may be related to the structure of the Notch3. Notch3 are single-pass transmembrane proteins consisting of extracellular, transmembrane, and intracellular domains. Upon activation, Notch is cleaved releasing the Notch intracellular domain (NICD) and NICD is then ready to be translocated into the nucleus for transcriptional activation of Notch target genes. Thus, the expression of Notch3 in nuclear will be less than the expression of Notch3 in cytoplasm and cell membrane. Nuclear Notch3 is an activator of Notch3 signalling pathway, so it may play an important role in function of Notch3 signalling pathway such as lymph node metastases. However, this result does not affect the correlation between Notch3 and pancreatic cancer. Similar results were also presented in other tissue. In hepatocellular carcinoma, Notch3 was detected in the cytoplasm (24). Positive staining of Notch3 was located in the cytoplasm and high Notch3 expression had a significantly shorter survival time, compared with those with no or low expression (25). Moreover, the results of multivariate analysis indicated the expression of Notch3 may be an indicator of worse outcome independent of TNM stage. The above-mentioned results suggest that a high expression of Notch3 is correlated with a worse patient outcome and may be an independent prognostic factor for PDAC. Moreover, in addition to TNM staging, Notch3 expression may be a useful prognostic biomarker for evaluating PDAC patients. This is the first report to show that the expression of Notch3 can be used as a prognostic biomarker for PDAC.
Many cancer patients die from metastasis, yet, the mechanism of metastasis remains unclear. To escape from the primary tumour and result in distant metastasis, tumour cells must acquire the ability to invade and migrate. Tumour cells from the primary tumour can be degraded and removed from the extracellular matrix and thus moved into the vicinity of the blood or lymphatic vessels, which lays the foundation for distant metastasis. Thus, to explore whether Notch3 plays an important role in PDAC, we focused on determining whether Notch3 may participate in the migration and invasion of PDAC in vitro.

For tumour cells to migrate, they first need to adhere to the blood or lymphatic vessels. Therefore, adhesion is an essential important process in the migration cascade. Many cell adhesion molecules, including integrins, cadherins, selectins, immunoglobulins and proteoglycans, have been implicated in tumour progression and metastasis. In the present study, we focused on CD44v6 and E-cadherin, which are two important adhesion receptors. CD44v6, a member of the CD44 family of cell adhesion molecules, plays an important role in the progression and metastasis of tumours (26). Previous research has shown that in many tumours, the abnormal expression of CD44v6 correlates with a poor prognosis $(27,28)$. E-cadherin is a main member of the ca-mucoprotein family, which is associated with differentiation and invasion of tumour cells (29). Numerous studies have indicated that E-cadherin plays an inhibitory role in tumour migration, metastasis and unfavourable prognosis (30-32). The reduction of E-cadherin expression and the degradation of E-cadherin adhesion plaques on the cell surface can result in cells escaping from the primary tumour and moving into the vicinity of the blood or lymphatic vessels (33). In the present study, it was interesting that inhibition of Notch3 decreased the migration of PDAC cells while decreasing the protein expression of CD44v6 and increasing the protein expression of E-cadherin. This finding indicated that Notch3 participates in the migration of PDAC cells and may regulate the expression of CD44v6 and E-cadherin.

However, how Notch3 regulates E-cadherin and CD44v6 is not clear. To further explore the potential mechanism governing how Notch3 regulates the expression of E-cadherin and CD44v6, we focused on COX-2, an upstream molecule of E-cadherin and CD44v6 (34,35). COX-2 participates in various cellular functions of tumours under physiologic and pathologic conditions (36,37). Abnormal expression of COX-2 also plays an important role in the progression of carcinogenesis $(34,38)$. During the progression of carcinogenesis, COX-2 contributes to the modulation of various molecules related to metastasis, such as E-cadherin and CD44v6 $(34,35)$. However, there are few studies concerning the correlation between the Notch signalling pathway and COX-2. In gastric cancer, N1IC, which is the activating factor of Notch1, can bind to a COX-2 promoter and thereby regulate the expression of COX-2 (39). However, whether Notch3 can regulate the expression of COX-2 is unclear. The results of the current experiments showed that inhibition of Notch3 reduces the expression of COX-2, indicating that Notch3 may be an upstream molecule of COX-2. However, the specific mechanisms of this process should be further explored. Our results indicate that Notch3 plays an important role in the process of migration in PDAC. However, many other mechanisms may be involved in the 
process of migration, and our results may highlight one of the possible mechanisms.

During the series of steps involved in tumour metastasis, tumour cells can degrade the basement membrane and the stromal extracellular matrix, which leads to tumour cell invasion. Matrix metalloproteinases (MMPs) and urokinase-type plasminogen activator (uPA) are important molecules involved in the process of invasion. MMPs are a family of related enzymes that can degrade the extracellular matrix (ECM) and cause tumour cells to invade the vasculature and target organs, leading to metastasis (40). MMPs, such as MMP-2 and MMP-9, can participate in the invasion and metastasis of the tumour, and they can degrade type IV collagen, which is the principal component of the basement membrane $(41,42)$. During cell migration, angiogenesis, tumour growth and metastasis, the plasminogen activator system plays an important role. uPA binds to its receptor (uPAR), which facilitates the conversion of plasminogen to plasmin. Plasmin participates in the invasion and metastasis of cancer cells by degrading components of the extracellular matrix, either directly or indirectly through MMPs (43).

VEGF plays an essential role in tumour cell invasion and metastasis. VEGF expression is commonly found to increase in tumours, and there is an association between VEGF expression and distant metastasis. Abnormal expression of VEGF increases the migration and invasion of tumour cells $(44,45)$. In the present study, we found that inhibition of Notch3 decreases the invasion of PDAC cells and reduce the expression of MMP-2, MMP-9, VEGF and uPA. Therefore, we can conclude that Notch3 participates in the invasion of PDAC cells by regulating the expression of MMP-2, MMP-9, VEGF and $\mathrm{UPA}$.

ERK1/2, a member of the family of mitogen-activated protein kinases (MAPKs), plays an important role in the signalling pathways related to scattering/motility, invasion, proliferation and survival $(46,47)$. In addition, activation of ERK1/2 regulates the expression of a variety of important genes in metastasis, including MMP-2/-9, VEGF and uPA $(48,49)$. Although in recent years, increased attention was paid to the interaction between the ERK1/2 pathway with other cell signal pathways, the relationship between the Notch signalling and the ERK1/2 pathways is unclear. The results showed that inhibition of Notch3 reduces the expression of pERK1/2, thereby inactivating the ERK1/2 pathway and regulating the expression of MMP-2/-9, VEGF and UPA. Based on the above-mentioned evidence, we suspect that this may be one of the mechanisms by which Notch3 participates in PDAC invasion.

In summary, our results strongly indicated that a high expression of Notch3 significantly correlated with the progression of PDAC and poor patient prognosis. Thus, Notch3 expression may be used as an adjunct to the TNM staging system to evaluate the prognosis of patients with PDAC. In vitro, inhibition of Notch3 can decrease the migration and invasion of PDAC cells by regulating the expression of CD44v6, E-cadherin, MMP-2, MMP-9, VEGF and uPA. Therefore, Notch3 may not be only a novel marker of prognosis for patients with PDAC but may also be a molecular target for PDAC therapy. However, the underlying mechanisms involved in these results should be further explored.

\section{Acknowledgements}

The present study was supported by grants from the National Natural Science Foundation of China (grants no. 81470843).

\section{References}

1. Jemal A, Siegel R, Xu J and Ward E: Cancer statistics, 2010. CA Cancer J Clin 60: 277-300, 2010.

2. Vincent A, Herman J, Schulick R, Hruban RH and Goggins M: Pancreatic cancer. Lancet 378: 607-620, 2011.

3. Hidalgo M: Pancreatic cancer. N Engl J Med 362: 1605-1617, 2010.

4. Stathis A and Moore MJ: Advanced pancreatic carcinoma: Current treatment and future challenges. Nat Rev Clin Oncol 7: 163-172, 2010.

5. Artavanis-Tsakonas S, Rand MD and Lake RJ: Notch signaling: Cell fate control and signal integration in development. Science 284: 770-776, 1999.

6. Miele L and Osborne B: Arbiter of differentiation and death: Notch signaling meets apoptosis. J Cell Physiol 181: 393-409, 1999.

7. Ohishi K, Katayama N, Shiku H, Varnum-Finney B and Bernstein ID: Notch signalling in hematopoiesis. Semin Cell Dev Biol 14: 143-150, 2003.

8. You L, Chen G and Zhao YP: Core signaling pathways and new therapeutic targets in pancreatic cancer. Chin Med J 123: 1210-1215, 2010.

9. De La O JP and Murtaugh LC: Notch signaling: Where pancreatic cancer and differentiation meet? Gastroenterology 136: 1499-1502, 2009.

10. Wang Z, Li Y and Sarkar FH: Notch signaling proteins: Legitimate targets for cancer therapy. Curr Protein Pept Sci 11: 398-408, 2010.

11. Wang Z, Zhang Y, Li Y, Banerjee S, Liao J and Sarkar FH: Down-regulation of Notch-1 contributes to cell growth inhibition and apoptosis in pancreatic cancer cells. Mol Cancer Ther 5: 483-493, 2006

12. Wang Z, Banerjee S, Li Y, Rahman KM, Zhang Y and Sarkar FH: Down-regulation of notch-1 inhibits invasion by inactivation of nuclear factor-kappaB, vascular endothelial growth factor, and matrix metalloproteinase-9 in pancreatic cancer cells. Cancer Res 66: 2778-2784, 2006.

13. Plentz R, Park JS, Rhim AD, Abravanel D, Hezel AF, Sharma SV, Gurumurthy S, Deshpande V, Kenific C, Settleman J, et al: Inhibition of gamma-secretase activity inhibits tumor progression in a mouse model of pancreatic ductal adenocarcinoma. Gastroenterology 136: 1741-1749.e6, 2009.

14. Ristorcelli E and Lombardo D: Targeting Notch signaling in pancreatic cancer. Expert Opin Ther Targets 14: 541-552, 2010.

15. Hanlon L, Avila JL, Demarest RM, Troutman S, Allen M, Ratti F, Rustgi AK, Stanger BZ, Radtke F, Adsay V, et al: Notch1 functions as a tumor suppressor in a model of $K$-ras-induced pancreatic ductal adenocarcinoma. Cancer Res 70: 4280-4286, 2010.

16. Chu D, Li Y, Wang W, Zhao Q, Li J, Lu Y, Li M, Dong G, Zhang H, Xie H, et al: High level of Notch1 protein is associated with poor overall survival in colorectal cancer. Ann Surg Oncol 17: 1337-1342, 2010.

17. Egan SE, St-Pierre B and Leow CC: Notch receptors, partners and regulators: From conserved domains to powerful functions. Curr Top Microbiol Immunol 228: 273-324, 1998.

18. Callahan R and Egan SE: Notch signaling in mammary development and oncogenesis. J Mammary Gland Biol Neoplasia 9: 145-163, 2004.

19. Mazur PK, Einwächter H, Lee M, Sipos B, Nakhai H, Rad R, Zimber-Strobl U, Strobl LJ, Radtke F, Klöppel G, et al: Notch2 is required for progression of pancreatic intraepithelial neoplasia and development of pancreatic ductal adenocarcinoma. Proc Natl Acad Sci USA 107: 13438-13443, 2010.

20. Yao J and Qian C: Inhibition of Notch3 enhances sensitivity to gemcitabine in pancreatic cancer through an inactivation of PI3K/Akt-dependent pathway. Med Oncol 27: 1017-1022, 2010.

21. Mullendore ME, Koorstra JB, Li YM, Offerhaus GJ, Fan X Henderson CM, Matsui W, Eberhart CG, Maitra A and Feldmann G: Ligand-dependent Notch signaling is involved in tumor initiation and tumor maintenance in pancreatic cancer. Clin Cancer Res 15: 2291-2301, 2009. 
22. Doucas H, Mann CD, Sutton CD, Garcea G, Neal CP, Berry DP and Manson MM: Expression of nuclear Notch3 in pancreatic adenocarcinomas is associated with adverse clinical features, and correlates with the expression of STAT3 and phosphorylated Akt. J Surg Oncol 97: 63-68, 2008.

23. Mann CD, Bastianpillai C, Neal CP, Masood MM, Jones DJ, Teichert F, Singh R, Karpova E, Berry DP and Manson MM: Notch3 and HEY-1 as prognostic biomarkers in pancreatic adenocarcinoma. PLoS One 7: e51119, 2012.

24. Gramantieri L, Giovannini C, Lanzi A, Chieco P, Ravaioli M, Venturi A, Grazi GL and Bolondi L: Aberrant Notch3 and Notch4 expression in human hepatocellular carcinoma. Liver Int 27: 997-1007, 2007.

25. Hu L, Xue F, Shao M, Deng A and Wei G: Aberrant expression of Notch3 predicts poor survival for hepatocellular carcinomas. Biosci Trends 7: 152-156, 2013.

26. Günthert U, Hofmann M, Rudy W, Reber S, Zöller M, Haussmann I, Matzku S, Wenzel A, Ponta H and Herrlich P: A new variant of glycoprotein CD44 confers metastatic potential to rat carcinoma cells. Cell 65: 13-24, 1991.

27. Jijiwa M, Demir H, Gupta S, Leung C, Joshi K, Orozco N, Huang T, Yildiz VO, Shibahara I, de Jesus JA, et al: CD44v6 regulates growth of brain tumor stem cells partially through the AKT-mediated pathway. PLoS One 6: e24217, 2011.

28. Liu YJ, Yan PS, Li J and Jia JF: Expression and significance of CD44s, CD44v6, and nm23 mRNA in human cancer. World J Gastroenterol 11: 6601-6606, 2005.

29. Kemler R: From cadherins to catenins: Cytoplasmic protein interactions and regulation of cell adhesion. Trends Genet 9: 317-321, 1993.

30. Bremnes RM, Veve R, Gabrielson E, Hirsch FR, Baron A, Bemis L, Gemmill RM, Drabkin HA and Franklin WA: High-throughput tissue microarray analysis used to evaluate biology and prognostic significance of the E-cadherin pathway in non-small-cell lung cancer. J Clin Oncol 20: 2417-2428, 2002

31. Tsujii $\mathrm{M}$ and DuBois RN: Alterations in cellular adhesion and apoptosis in epithelial cells overexpressing prostaglandin endoperoxide synthase 2. Cell 83: 493-501, 1995.

32. Liu D, Huang C, Kameyama K, Hayashi E, Yamauchi A Kobayashi S and Yokomise: E-cadherin expression associated with differentiation and prognosis in patients with non-small cell lung cancer. Ann Thorac Surg 71: 949-954, 2001.

33. Wells A, Yates C and Shepard CR: E-cadherin as an indicator of mesenchymal to epithelial reverting transitions during the metastatic seeding of disseminated carcinomas. Clin Exp Metastasis 25: 621-628, 2008.

34. Dohadwala M, Batra RK, Luo J, Lin Y, Krysan K, Pold M, Sharma S and Dubinett SM: Autocrine/paracrine prostaglandin E2 production by non-small cell lung cancer cells regulates matrix metalloproteinase-2 and CD44 in cyclooxygenase-2-dependent invasion. J Biol Chem 277: 50828-50833, 2002.

35. Dohadwala M, Yang SC, Luo J, Sharma S, Batra RK, Huang M, Lin Y, Goodglick L, Krysan K, Fishbein MC, et al: Cyclooxygenase-2-dependent regulation of E-cadherin: Prostaglandin $\mathrm{E}_{2}$ induces transcriptional repressors ZEB1 and snail in non-small cell lung cancer. Cancer Res 66: 5338-5345, 2006.
36. Dubinett SM, Sharma S, Huang M, Dohadwala M, Pold M and Mao JT: Cyclooxygenase-2 in lung cancer. Prog Exp Tumor Res 37: 138-162, 2003.

37. Dannenberg AJ and Subbaramaiah K: Targeting cyclooxygenase-2 in human neoplasia: Rationale and promise. Cancer Cell 4: 431-436, 2003.

38. Park K, Han S, Shin E, Kim HJ and Kim JY: Cox-2 expression on tissue microarray of breast cancer. Eur J Surg Oncol 32: 1093-1096, 2006

39. Yeh TS, Wu CW, Hsu KW, Liao WJ, Yang MC, Li AF, Wang AM, Kuo ML and Chi CW: The activated Notch1 signal pathway is associated with gastric cancer progression through cyclooxygenase-2. Cancer Res 69: 5039-5048, 2009.

40. Itoh $\mathrm{Y}$ and Nagase $\mathrm{H}$ : Matrix metalloproteinases in cancer. Essays Biochem 38: 21-36, 2002.

41. Zeng ZS, Cohen AM and Guillem JG: Loss of basement membrane type IV collagen is associated with increased expression of metalloproteinases 2 and 9 (MMP-2 and MMP-9) during human colorectal tumorigenesis. Carcinogenesis 20: 749-755, 1999.

42. Komatsu K, Nakanishi Y, Nemoto N, Hori T, Sawada T and Kobayashi M: Expression and quantitative analysis of matrix metalloproteinase-2 and -9 in human gliomas. Brain Tumor Pathol 21: 105-112, 2004.

43. Shimizu M, Cohen B, Goldvasser P, Berman H, Virtanen C and Reedijk M: Plasminogen activator $u P A$ is a direct transcriptional target of the JAG1-Notch receptor signaling pathway in breast cancer. Cancer Res 71: 277-286, 2011.

44. Wey JS, Fan F, Gray MJ, Bauer TW, McCarty MF, Somcio R, Liu W, Evans DB, Wu Y, Hicklin DJ, et al: Vascular endothelial growth factor receptor-1 promotes migration and invasion in pancreatic carcinoma cell lines. Cancer 104: 427-438, 2005.

45. Takahashi Y, Kitadai Y, Bucana CD, Cleary KR and Ellis LM Expression of vascular endothelial growth factor and its receptor, KDR, correlates with vascularity, metastasis, and proliferation of human colon cancer. Cancer Res 55: 3964-3968, 1995.

46. Chan-Hui PY and Weaver R: Human mitogen-activated protein kinase kinase kinase mediates the stress-induced activation of mitogen-activated protein kinase cascades. Biochem J 336: 599-609, 1998

47. Trusolino L and Comoglio PM: Scatter-factor and semaphorin receptors: Cell signalling for invasive growth. Nat Rev Cancer 2: 289-300, 2002

48. Arai K, Lee SR and Lo EH: Essential role for ERK mitogenactivated protein kinase in matrix metalloproteinase-9 regulation in rat cortical astrocytes. Glia 43: 254-264, 2003.

49. Cheng YC, Chen LM, Chang MH, Chen WK, Tsai FJ, Tsai CH, Lai TY, Kuo WW, Huang CY and Liu CJ: Lipopolysaccharide upregulates uPA, MMP-2 and MMP-9 via ERK1/2 signaling in H9c2 cardiomyoblast cells. Mol Cell Biochem 325: 15-23, 2009. 